\title{
Síndrome de piel indurada en un paciente pediátrico: un desafío terapéutico. Presentación de un caso
} Stiff skin syndrome in a pediatric patient: a therapeutic challenge. Clinical case

\author{
Dra. Montserrat Colombarolli ${ }^{a}$ Dr. Alejandro Olivera ${ }^{a}$, Dra. Mariángeles Domíngueza ${ }^{a}$ Dra. Ana Cirio ${ }^{a}$ \\ Dra. Carla Castro y Dra. Corina Busso ${ }^{a}$
}

\section{RESUMEN}

El síndrome de la piel indurada es un trastorno esclerosante crónico, infrecuente, que se presenta en la infancia, caracterizado por la induración progresiva de la piel. Esta afección puede provocar restricciones torácicas y dificultad respiratoria, limitaciones en la movilidad articular y trastornos en la marcha, con importante deterioro de la calidad de vida. Debido a que sus opciones terapéuticas son escasas y poco eficaces, es fundamental que el paciente inicie precozmente una terapia física para prevenir estas complicaciones y que se continúe estudiando esta enfermedad a fin de poder ofrecer a los pacientes más y mejores tratamientos. Se presenta el caso de una paciente de 9 años con síndrome de la piel indurada y su desafío terapéutico.

Palabras clave: enfermedades de la piel y del tejido conectivo, sindrome de la piel indurada, losartán.

\begin{abstract}
Stiff skin syndrome is a chronic, rare sclerosing disorder that occurs in childhood, characterized by progressive induration of the skin that can cause thoracic restrictions and respiratory distress, limitations in joint mobility and gait difficulties, with significant deterioration of the quality of life. Because their therapeutic options are scarce and ineffective it is essential to start an early physical therapy to prevent these complications and to continue studying this condition to be able to offer patients more and better treatments. We present the case of a 9-year-old patient with indurated skin syndrome and its therapeutic challenge.
\end{abstract}

Key words: skin and connective tissue diseases, stiff skin syndrome, losartan.

http: / / dx.doi.org/10.5546/ aap.2020.e463

Cómo citar: Colombarolli M, Olivera A, Domínguez M, Cirio A, et al. Síndrome de piel indurada en un paciente pediátrico: un desafío terapéutico. Presentación de un caso. Arch Argent Pediatr 2020;118(5):e463-e467.

a. Servicio de Dermatología, Hospital Universitario Austral, Universidad Austral, Pilar, provincia de Buenos Aires, Argentina.

Correspondencia:

Dra. Montserrat Colombarolli: mcolomba@cas.austral.edu.ar

Financiamiento: Ninguno.

Conflicto de intereses: Ninguno que declarar.

Recibido: 12-11-2019

Aceptado: $16-3-2020$

\section{INTRODUCCIÓN}

El síndrome de la piel indurada es un desorden no inflamatorio del tejido conectivo, esclerodermiforme, que fue descrito en 1971 por Esterly y Mc Kusik. ${ }^{1}$ Se trata de una patología infrecuente, con 58 casos publicados en la literatura mundial. Es poco lo que se conoce con respecto a la evolución de los pacientes, y las opciones terapéuticas descritas son desalentadoras. ${ }^{2-4}$

Se presenta a una paciente pediátrica con criterios clínicos e histopatológicos de síndrome de la piel indurada. Se realizó una revisión de la literatura, con especial énfasis en las opciones terapéuticas actuales.

\section{CASO CLÍNICO}

Una paciente de sexo femenino, de 9 años de edad, sin antecedentes familiares de importancia, fue traída a la consulta por presentar piel indurada en los miembros inferiores y la cintura pelviana, lo que dificultaba su movilidad para realizar las actividades habituales. Esta característica de la piel comenzó a manifestarse durante los primeros meses de vida y fue progresando a lo largo del tiempo.

A los 3 años de edad, fue evaluada en otro hospital, donde se le realizó una ecografía de partes blandas, que describió, a nivel del tercio proximal de ambos muslos, un engrosamiento del tejido celular subcutáneo, y una biopsia de piel, compatible con nevo de tejido conectivo. La paciente asistía a la escuela, tenía un desarrollo madurativo acorde con su edad y practicaba gimnasia deportiva desde muy pequeña con dificultad.

En el examen físico, se observó una piel de aspecto acartonado en ambos muslos, los glúteos, las piernas y el área lumbosacra. En el dorso de ambos muslos, se visualizaron aisladas y pequeñas máculas hiperpigmentadas e hipopigmentadas con límites difusos sobre la misma piel comprometida, así como un pequeño parche de hipertricosis en la cara lateral del muslo 
derecho y en el área lumbosacra (Figuras 1 y 2). La piel se palpaba indurada, con límites poco definidos y con dificultad para plegarse.

A nivel osteoarticular, se observó una marcada hiperlordosis y asimetría de los miembros inferiores, que ocasionaba una ligera escoliosis. Además, la paciente presentaba alteración en la movilidad articular de la cadera y las rodillas, que le ocasionaba dificultad para extender los miembros inferiores y deambular con normalidad (Figura 3).

Los estudios de laboratorio de rutina, que incluyeron anticuerpos antinucleares y complemento, fueron normales. La radiografía de columna, junto con la resonancia magnética nuclear de la pelvis y la columna, informó una leve rectificación de la lordosis fisiológica cervical y desviación dorsolumbar de convexidad izquierda.

La biopsia del muslo derecho mostró, en la dermis reticular profunda y tabiques hipodérmicos, esclerosis con engrosamiento de haces de colágeno, varios de ellos de disposición horizontal, atrapamiento focal de adipocitos

FIGURA 1. Hipo- e hiperpigmentación en el tercio proximal de los muslos sobre la piel afectada. Parche de hipertricosis en la cara lateral del tercio proximal del muslo derecho

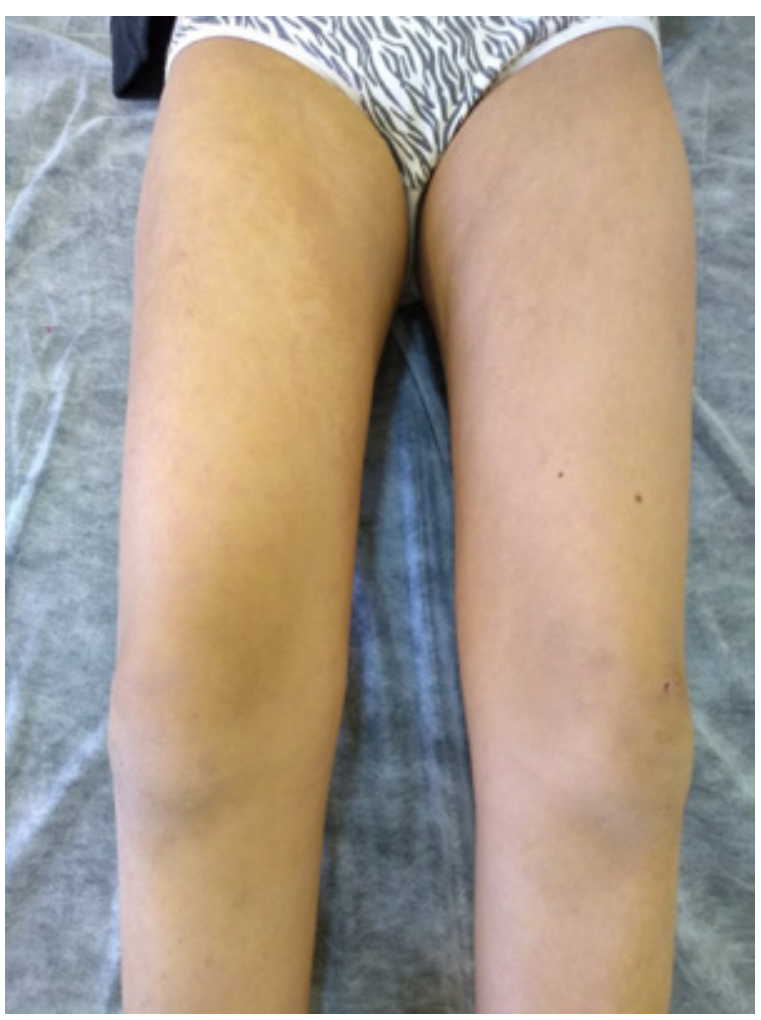

en la dermis reticular profunda, sin signos de inflamación. La técnica de Alcian Blue evidenció un leve incremento de mucopolisacáridos intersticiales focales. El estudio histopatológico de la piel sana contralateral fue normal (Figura 4).

A partir de los datos obtenidos del examen clínico y del estudio histopatológico, se arribó al diagnóstico de síndrome de piel indurada. Aún está pendiente el estudio de la mutación del gen fibrilina 1 (FBN1).

Se explicó a la paciente y a su familia que era una patología poco frecuente y, luego de una revisión bibliográfica, se plantearon como opciones terapéuticas drogas con actividad antifibrótica, como el losartán o el micofenolato mofetil. Debido a que ambas eran de uso off label, se firmó un consentimiento informado. Inicialmente, se indicó kinesioterapia y ejercicio físico, además de tratamiento oral con losartán, administrado una vez por día en una dosis inicial de $0,4 \mathrm{mg} / \mathrm{kg}$, con controles de tensión arterial y frecuencia cardíaca, que fueron normales. A la semana, se aumentó a $0,7 \mathrm{mg} / \mathrm{kg} / \mathrm{d}(25 \mathrm{mg}$ diarios), con buena tolerancia.

FIGURA 2. Hipertricosis e induración de la zona lumbosacra; trayecto fibroso hiperpigmentado en el lateral derecho

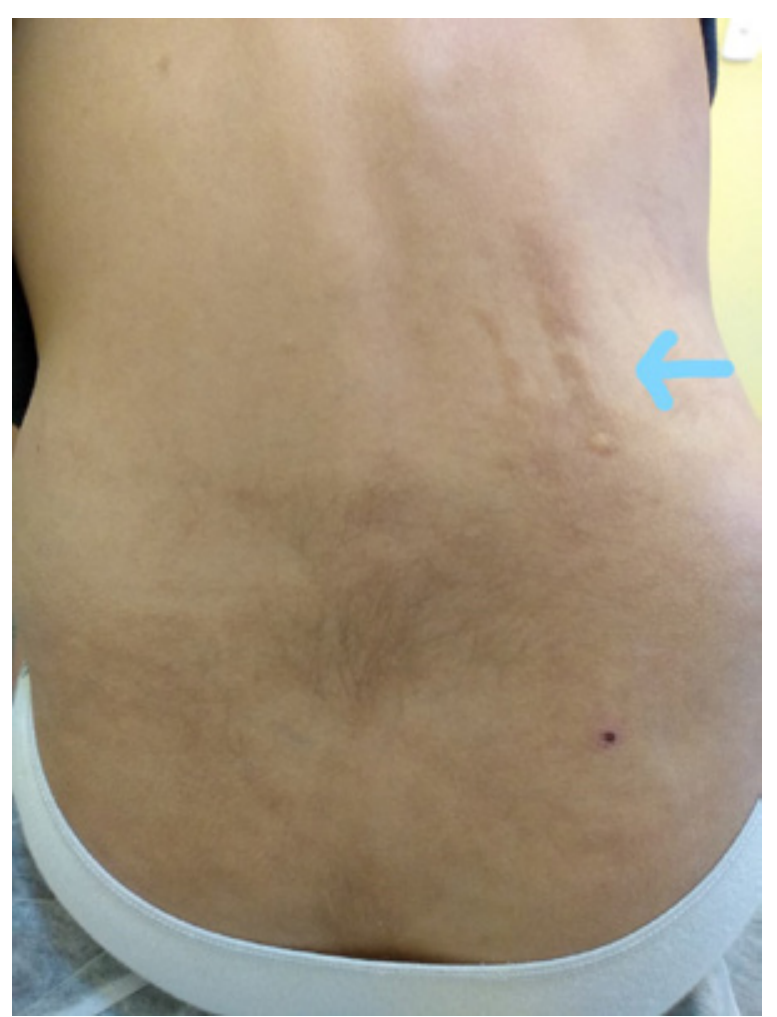


FIGURA 3. Asimetría en la cintura escapular y pelviana. Ligera escoliosis. Dificultad para extender los miembros inferiores, con predominio derecho

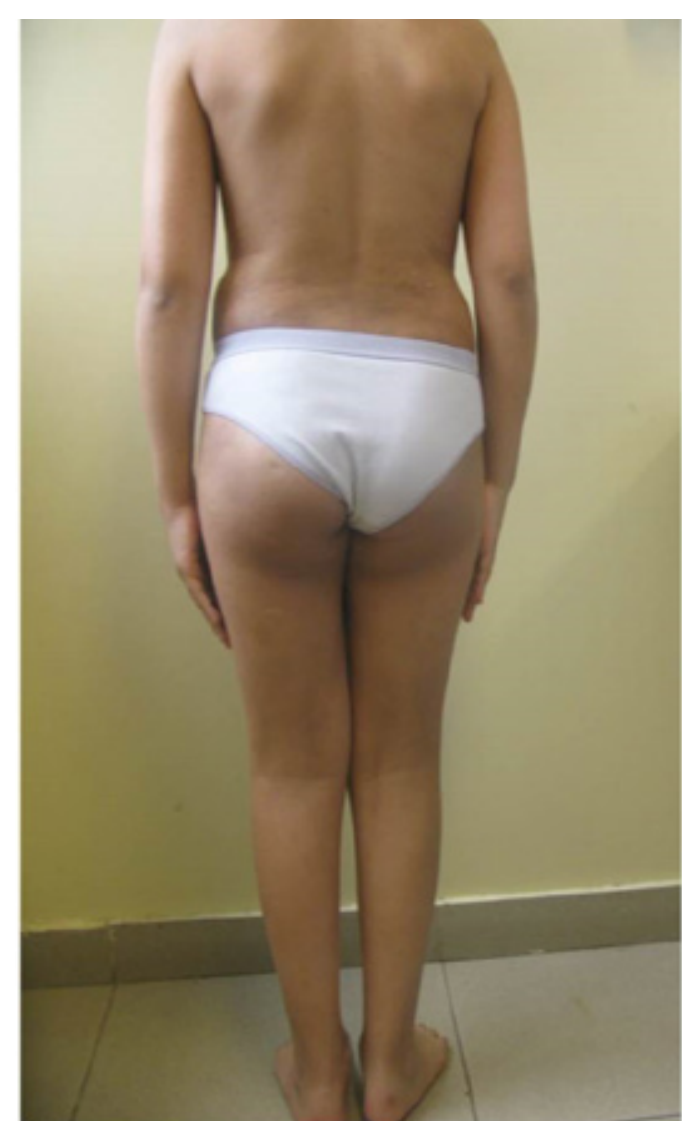

La respuesta terapéutica se evaluó con la escala Physician's Global Assessment (PGA): $0=\sin$ induración; 1 = induración leve, en la que la piel podía pellizcarse fácilmente; 2 = induración moderada, en la que la piel podía pellizcarse con presión; 3 = induración de moderada a grave, en la que la piel podía pellizcarse con mucha presión; 4 = induración grave, con la piel duro-pétrea imposible de pellizcar. La paciente discontinuó el tratamiento farmacológico a los dos meses por falta de respuesta, razón por la cual no se pudo evaluar su efectividad a largo plazo. Con una puntuación inicial de 3, no presentó ningún signo de mejoría.

Si bien el compromiso de su piel era significativo, la niña podía desarrollar sus actividades diarias sin mayores restricciones. Continuaba con kinesioterapia, magnetoterapia y reeducación postural global (RPG) 2 veces por semana, además de practicar yoga con ejercicios de elongación. Se decidió, en conjunto con sus padres, que retomara el tratamiento sistémico en caso de que la enfermedad progresara.

\section{DISCUSIÓN}

El síndrome de la piel indurada es un desorden genético asociado a una mutación del gen fibrilina 1, localizado en el cromosoma 15 (FBN1/Cr15), que, en condiciones normales, codifica la proteína fibrilina 1, la cual limita la

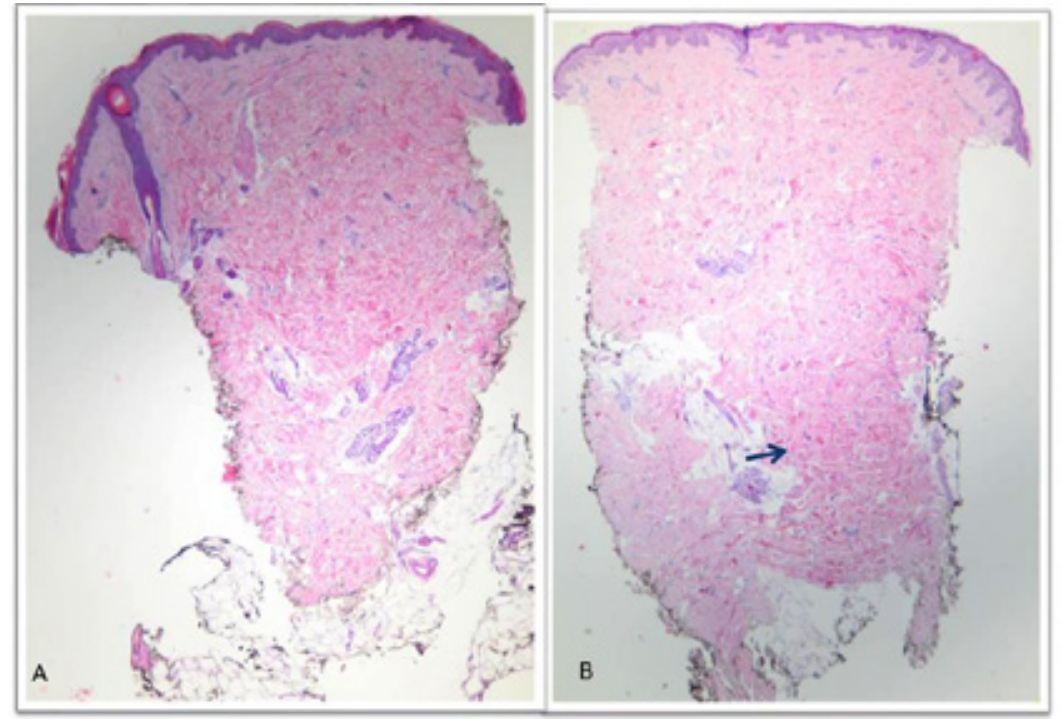

Evaluada por el doctor Javier Anaya, patólogo. A: Piel sana de la cara lateral del muslo izquierdo. B: Piel afectada de la cara lateral del muslo derecho, con fibras de colágeno engrosadas y atrapamiento de adipocitos maduros en la dermis reticular profunda. 
activación y señalización del factor de crecimiento transformante beta (transforming growth factorbeta; TGF- $B$, por sus siglas en inglés), una citocina profibrótica. La alteración del gen fibrilina 1 genera una actividad profibrótica, con la consiguiente acumulación de colágeno VI. ${ }^{5}$ En un $20 \%$ de los casos, se hereda de forma autosómica dominante. $^{2}$

La mayoría de los casos documentados son pediátricos. Hay pocos casos descritos en adultos; todos ellos, jóvenes. Llama la atención el reporte de un paciente de 46 años, lo cual podría deberse a una remisión parcial o total del cuadro a lo largo de la vida o a un subdiagnóstico en virtud del desconocimiento de la entidad. ${ }^{6,7}$

Clínicamente, el síndrome de piel indurada se manifiesta al nacer o en la infancia temprana, con la instalación progresiva de la induración de la piel. Su curso es lento y va comprometiendo las áreas donde abunda la fascia muscular, como los hombros y la cintura pelviana. Se describe con más frecuencia en los glúteos, los muslos y el área lumbar, tal como se manifiesta en este caso. La apariencia de la piel puede ser normal o asociarse a hipertricosis y cambios en la pigmentación. ${ }^{3} \mathrm{El}$ compromiso, generalmente, es simétrico, aunque, en 2016, Myers y col., describieron la variante clínica segmentaria, de inicio más tardío, menos grave. $^{8}$

Las características extracutáneas incluyen contracturas articulares, escoliosis y dificultades en la deambulación. Esta entidad no presenta compromiso muscular, óseo ni visceral. ${ }^{3}$

La histopatología, que no es confirmatoria, pero orienta el diagnóstico, se caracteriza por la presencia de haces de colágeno engrosados con orientación horizontal, ausencia de inflamación, depósito de mucina variable en la dermis y atrapamiento de adipocitos, con el eventual engrosamiento de la fascia. ${ }^{2,9}$ Los diagnósticos diferenciales incluyen la esclerodermia sistémica, la morfea, el nevo de tejido conectivo y otros cuadros esclerodermiformes. ${ }^{3}$

Debido a que es un síndrome muy poco frecuente, no hay guías de tratamiento, y las opciones terapéuticas reportadas, como inmunosupresores o antiinflamatorios, son de uso off label y arrojan resultados desalentadores: calcipotriol o corticoides tópicos y terapéuticas sistémicas con pulsos de metilprednisolona, metotrexato, terapia PUVA, penicilamina, ciclosporina, micofenolato mofetil y losartán. ${ }^{3}$ Kurtzman y otros reportaron a dos pacientes de 25 y 32 años, respectivamente, que recibieron de
1 a 1,5 g de micofenolato mofetil, 2 veces por día, durante 3-6 meses, con mejoría leve y recurrencia al finalizar el tratamiento. ${ }^{6}$ Maillet-Lebel y otros refirieron una mejoría parcial en un paciente de 6 años, que realizó tratamiento con losartán a razón de $0,7 \mathrm{mg} / \mathrm{kg} / \mathrm{d}(50 \mathrm{mg} / \mathrm{d})$ durante 2 años. ${ }^{2}$ Ambas drogas tendrían su efecto antifibrótico mediante la disminución de la señalización del TGF-B. ${ }^{4}$

La dosis máxima de losartán que recibió nuestra paciente fue de $25 \mathrm{mg} /$ día con buena tolerancia, pero sin signos de mejoría. Otra terapéutica interesante, pero que aún se encuentra en la fase de investigación en ratones, son los moduladores de la integrina, que previenen la fibrosis en la piel, y los antagonistas del TGF-B, que la revierten. ${ }^{10}$

El pronóstico y la evolución son poco conocidos, debido a que se trata de pacientes pediátricos en la mayoría de los casos reportados. El síndrome de la piel indurada es una dermatosis crónica símil esclerodermia de inicio temprano en la niñez, que se acompaña de trastornos en la movilidad articular, hipertricosis y cambios posturales. Se reportó el caso de una niña que presentaba el síndrome de la piel indurada, con clínica e histopatología clásicas, sin antecedentes familiares. Se planteó el desafío que representaba la terapéutica, con el objetivo de mejorar la calidad de vida de estos pacientes y evitar comorbilidades. Es necesario conocer más y mejor esta entidad, a fin de poder brindar al paciente y a su familia más información sobre su pronóstico y mejores opciones terapéuticas.

\section{REFERENCIAS}

1. Esterly NB, McKusick VA. Stiff skin syndrome. Pediatrics. 1971; 4(2):360-9.

2. Maillet-Lebel N, Kokta V, Coulombe J, Powell J. A case of segmental stiff skin syndrome treated with systemic losartan. Pediatr Dermatol. 2018; 35(1):e66-7.

3. Liu T, McCalmont TH, Frieden IJ, Williams ML, et al. The stiff skin syndrome: case series, differential diagnosis of the stiff skin phenotype and review of the literature. Arch Dermatol. 2008; 144(10):1351-9.

4. Deng F, Chen Z, Song H, Yan Y, et al. Four new cases of stiff skin syndrome with unusual presentations. J Eur Acad Dermatol Venereol. 2016; 30(1):163-5.

5. Jablonska S, Groniowski J, Krieg T, Nerlich A, et al. Congenital fascial dystrophy-a noninflammatory disease of fascia: the stiff skin syndrome. Pediatr Dermatol. 1984; 2(2):87-97.

6. Kurtzman DJB, Wright NA, Patel M, Vleugels RA. Segmental stiff skin syndrome: Two additional cases with a positive response to mycophenolate mofetil and physical therapy. J Am Acad Dermatol. 2016; 75(6):e237-9.

7. Sari A, Cirakoglu D. Middle-aged female diagnosed with widespread stiff skin syndrome. Arch Rheumatol. 2018; 33(4):491-3. 
8. Myers K, Mir A, Schaffer J, Meehan SA, et al. Segmental stiff skin syndrome: a distinct clinical entity. J Am Acad Dermatol. 2016; 75(1):163-8.

9. Bachour J, Ghandour F, Ammoury A. Stony hard skin during early infancy. Am J Dermatopathol. 2018;40(8):614-6.
10. Gerber EE, Gallo EM, Fontana SC, Davis EC, et al. Integrin-modulating therapy prevents fibrosis and autoimmunity in mouse models of scleroderma. Nature. 2013; 503(7474):126-30. 